\title{
Hospital-based incidence of maternal heart failure during pregnancy in Nigeria
}

This article was published in the following Dove Press journal:

International Journal of General Medicine

5 April 2013

Number of times this article has been viewed

\author{
Patience Olayinka Akinwusi ${ }^{1,2}$ \\ Adetunji Oladeni Adeniji ${ }^{3}$ \\ Oluseyi Olaboyede Atanda ${ }^{3}$ \\ Adebayo Duyile Adekunle ${ }^{3}$ \\ 'Department of Medicine, College \\ of Health Sciences, Osun State \\ University, ${ }^{2}$ Department of Medicine, \\ ${ }^{3}$ Department of Obstetrics and \\ Gynecology, Ladoke Akintola \\ University of Technology Teaching \\ Hospital, Osogbo, Nigeria
}

Purpose: To determine the incidence of heart failure during pregnancy and incriminated cardiac lesions, as well as maternal and fetal outcomes in Ladoke Akintola University of Technology Teaching Hospital (LTH), Osogbo, Southwest Nigeria.

Methods: This study was a retrospective, descriptive review of all cases of heart failure during pregnancy based on data retrieved from the medical records of LTH over a 7-year period from January 2004 to December 2010. Analysis of these data was carried out using SPSS 17. Ethical clearance was obtained from the Ethical Committee of LTH.

Results: A total of 4523 patients delivered babies over the 7-year period, ten (2.2/1000) of which had cardiac decompensation. All patients were not registered at LTH for antenatal care (unbooked), with $70 \%$ of them aged 18-24 years. Fifty percent were primigravidae and the majority of them presented with symptoms in the second and third trimester. There were two cases of maternal deaths recorded and three cases of fetal/perinatal mortality. Only one case of congenital heart disease (pulmonary stenosis) and no cases of rheumatic heart disease were found.

Conclusion: The data suggests that heart failure during pregnancy is uncommon in Southwest Nigeria. However, it occurs more often in young, unbooked primigravid women. Efforts should be aimed at encouraging early booking for antenatal care and a full cardiovascular evaluation to prevent associated maternal and fetal/perinatal morbidity and mortality.

Keywords: heart failure, pregnancy, cardiac disease, fetal outcome, maternal outcome

\section{Introduction}

Cardiac disease during pregnancy has been shown to be associated with maternal and neonatal complications in developed countries despite the advancement in medical care. ${ }^{1-5}$ These complications often translate to high maternal and fetal/perinatal morbidity and mortality especially in undeveloped countries. ${ }^{6}$ Various studies have shown the incidence of cardiac disease during pregnancy to be $0.1 \%-4.0 \%{ }^{7-10}$ The pregnancy-related physiological changes of increased blood volume, heart rate, and cardiac output - especially in the second half of pregnancy - coupled with the stress of labor and the hemodynamic changes of the puerperium may aggravate an underlying cardiac disease in an otherwise clinically stable woman. ${ }^{11,12}$ Pregnant women with cardiac disease may go into either right- or left-sided heart failure, which may be precipitated or intensified by a lengthy list of extra cardiac factors including pregnancy itself. ${ }^{13}$ In developed countries, congenital heart disease (CHD) is the most common cause of cardiac disease, accounting for $89 \% ;{ }^{14}$ whereas rheumatic heart disease (RHD) constitutes the major cause in developing countries. ${ }^{15}$
Akinwusi

Department of Medicine, College of Health Sciences, Osun State University, Oke-Baale, PMB 4494, Osogbo,

Osun State, Nigeria

Tel +23480372I 945

Email pat.akinwusi@uniosun.edu.ng 
In sub-Saharan Africa, there is now a double burden of poverty-related cardiac disease (eg, RHD) and chronic noncommunicable diseases (eg, hypertension) ${ }^{16}$ Furthermore, there has been a four-fold rise in maternal mortality in South Africa over the past decade due, in part, to their poor health care system; heart disease has been found to account for $41 \%$ of the indirect causes of maternal deaths. ${ }^{17}$ Data of these nature are lacking in our setting in Nigeria.

This study, therefore, embarked on finding out the hospital-based incidence of heart failure during pregnancy and incriminated cardiac lesions, as well as maternal mortality and fetal/neonatal outcomes in a Nigerian tertiary health facility.

\section{Patients and methods}

The study was a descriptive, retrospective review of case notes of all booked and unbooked pregnant women who delivered at Ladoke Akintola University of Technology Teaching Hospital (LTH), Osogbo, Southwest Nigeria within the 7-year period of January 2004 to December 2010. All of the women who presented with cardiac decompensation during pregnancy were selected and information on demographic data, New York Heart Association (NYHA) class, investigations, treatment, and maternal and fetal/neonatal outcomes was then retrieved from their case notes. Investigation results retrieved from the case notes included twelve-lead resting electrocardiogram, echocardiogram, chest X-ray with shielded abdomen in a few of the patients, electrolytes, urea and creatinine, urinalysis, full blood count, hemoglobin genotype, and blood culture in those with sepsis.

Patients with hypertension who were not in heart failure, diabetic patients, and those with preeclampsia or eclampsia were excluded. Only those with hypertensive heart disease were included, which included patients with hypertensive heart failure. Ethical clearance was obtained from the Ethical Committee of LTH.

Statistical analysis was performed with SPSS Statistics 17 (SPSS Inc, Chicago, IL, USA). Results are presented in frequencies and percentages.

\section{Results}

The total number of obstetric cases during the 7-year period was 4523; of which, only ten reported cardiac decompensation, ie, an incidence of 2.2/1000. All the patients were unbooked for antenatal care at LTH.

Table 1 shows the demographic and clinical data of the patients, the etiology of heart failure, and maternal mortality and fetal/neonatal outcome. Seventy percent of the patients
Table I Heart failure during pregnancy: demography, etiology of heart failure, neonatal outcome, and maternal mortality $(n=10)$

\begin{tabular}{|c|c|c|c|}
\hline Characteristics & $\begin{array}{l}\text { Number } \\
\text { of patients, } \\
\text { n (\%) }\end{array}$ & $\begin{array}{l}\text { Neonatal } \\
\text { outcome }\end{array}$ & $\begin{array}{l}\text { Maternal } \\
\text { mortality }\end{array}$ \\
\hline \multicolumn{4}{|l|}{ Age range, years } \\
\hline $18-24$ & $7(70)$ & 3 (dead) & 2 \\
\hline $25-34$ & 0 & 0 & 0 \\
\hline $35-40$ & $3(30)$ & 0 & 0 \\
\hline \multicolumn{4}{|l|}{ Parity } \\
\hline Primipara/primigravida & $5(50)$ & 3 (dead) & 2 \\
\hline Secondary & $2(20)$ & 0 & 0 \\
\hline Multipara & $3(30)$ & 0 & 0 \\
\hline \multicolumn{4}{|l|}{ EGA at presentation } \\
\hline First trimester & 0 & 0 & 0 \\
\hline Second trimester & $3(30)$ & 3 (dead) & 2 \\
\hline Third trimester & $5(50)$ & 0 & 0 \\
\hline Post-term & I (10) & 0 & 0 \\
\hline Postdelivery & I (10) & 0 & 0 \\
\hline \multicolumn{4}{|l|}{ NYHA class } \\
\hline \multicolumn{4}{|l|}{ I } \\
\hline \multicolumn{4}{|l|}{$\|$} \\
\hline III & $5(50)$ & 0 & 0 \\
\hline IV & $5(50)$ & 3 (dead) & 2 \\
\hline \multicolumn{4}{|l|}{ Etiology of HF } \\
\hline HB with sepsis & 2 & 2 (FSB) & 2 \\
\hline $\mathrm{HHD}$ & 7 & $\begin{array}{l}6 \text { (alive, } \\
\text { I DAMA) }\end{array}$ & 0 \\
\hline Pulmonary stenosis & I & I (EPD) & 0 \\
\hline
\end{tabular}

Abbreviations: EGA, estimated gestational age; EPD, early perinatal death; DAMA, discharge against medical advice; $\mathrm{FSB}$, fresh still birth; $\mathrm{HB}$, hemoglobinopathy; $\mathrm{HF}$, heart failure; HHD, hypertensive heart disease; NYHA, New York Heart Association.

were aged $18-24$ years. The majority of the patients $(80 \%)$ presented in the second and third trimester. Seventy percent of the patients had hypertensive heart failure with blood pressure ranging from $128 / 90$ to $180 / 120$. There was only one case $(10 \%)$ of CHD. This was a case of pulmonary stenosis with sepsis syndrome, and the neonatal outcome was early perinatal death. The two patients with hemoglobinopathy who presented with heart failure had a hemoglobin concentration of less than $4 \mathrm{~g} / \mathrm{L}$, associated sepsis syndrome, and were NYHA class IV.

In addition to dyspnea as stated by the NYHA classification of the patients, the patients had other symptoms and signs of heart failure such as cough, bilateral pitting pedal edema, elevated jugular venous pressure, S3 gallop rhythm, and tender hepatomegaly. Bibasal crepitations were present in $80 \%$ of the patients, $10 \%$ had widespread crepitations in both lung fields with occasional rhonchi, and the remaining $10 \%$ (the only case of pulmonary stenosis) had bronchovesicular breath sounds without crepitations. The patient with pulmonary stenosis had functional tricuspid regurgitation murmur. 
All of the patients except one, who discharged herself against medical advice, had spontaneous vertex delivery within a few days of presentation at the hospital. All of the patients were human immunodeficiency virus negative.

The results were as expected for patients with heart failure and the specific cardiac lesions shown in Table 2. The pulmonary stenosis was severe, as demonstrated by a pressure gradient of $96 \mathrm{mmHg}$ across the pulmonary valve. The blood cultures of the patients with sepsis did not grow any organisms, which is not uncommon in the authors' practice area as patients are likely to have been given antibiotics and other drugs before reporting to the hospital, hence empirical antibiotics were given. Some investigations were not carried out due to financial constraints or due to a very short duration of stay in the hospital before the patient died (as retrieved from the case notes).

Table 3 shows the treatment given to each of the ten patients. All patients had intravenous furosemide and intranasal oxygen and three of them had spironolactone. Other treatments were individualized based on the underlying cardiac lesion responsible for the heart failure. Intravenous hydralazine and oral $\alpha$-methyldopa were given as appropriate to those in hypertensive heart failure to control their blood pressure. Digoxin was given to four of the patients with hypertensive heart failure who had poor systolic function on echocardiography. Antibiotics and antimalarials were given to those who had clinical and objective laboratory evidence of infection and malaria, respectively.

\section{Discussion}

The incidence of heart failure during pregnancy was 2.2/1000 in this study. However, there is a lack of data on this subject, hence the reference to cardiac disease during pregnancy, in which incidences of $0.1 \%-4.0 \%$ have been reported in other centers. In a study in sub-Saharan Africa, Diao et al found that $72 \%$ of the pregnant women with heart disease presented with heart failure. ${ }^{18}$ It is also noteworthy that no cases of RHD as a cause of heart failure during pregnancy and only one case of CHD, ie, pulmonary stenosis, were found in the current study. This may be due to the fact that both RHD and CHD are uncommon, as shown in a recently concluded 8-year prospective echocardiographic evaluation of 2501 patients presenting in one of the authors' teaching hospitals, located in the same state as LTH. ${ }^{19}$ The prevalence of RHD and CHD was 3.1\% and 2.1\%, respectively. ${ }^{19}$ At the Federal Medical Center, Abeokuta, Southwest Nigeria, an echocardiography study by Ogah et al reported 3.7\% cases of RHD out of the 1441 subjects studied. ${ }^{20}$ Even in the Nigerian Savannah, where Sani et al reported a relatively higher prevalence of $9.8 \%$ from echocardiography screening about 5 years ago, the prevalence of RHD was still much lower than that of South Africa. ${ }^{21}$ Also, the majority of patients with RHD and CHD die in their childhood or as adolescents and never reach the reproductive age group when they can present with cardiac disease during pregnancy. The tertiary health care system lacks the appropriate facilities for open heart surgery to perform the needed corrections for these cardiac lesions, and treatment abroad is beyond the reach of most of these patients. RHD has been reported to be the most common cause of cardiac disease during pregnancy in resource poor countries. ${ }^{15}$ This is particularly so in South Africa, with a high incidence of 36\% reported for RHD in the Heart of Soweto Study. ${ }^{16}$ Nqayana et al reported RHD in $81 \%(\mathrm{n}=77)$ of the 95 patients presenting with cardiac disease during pregnancy over a 1-year period at Inkosi Albert Luthuli Central Hospital, Durban, South Africa. ${ }^{6}$ Similarly, a 4-year audit of cardiac disease during pregnancy at Pretoria Academic Hospital showed RHD as the etiology in 63.5\% of the patients, while prosthetic valvular heart disease was $20.1 \%$ with also a possible etiology of RHD. ${ }^{11}$

There is now better control of communicable disease in Nigeria as the health care delivery system is better equipped to tackle such problems. Advocacy as well as continuous and intense health education are being used to fight communicable disease in Nigeria. Solid primary health care now forms the bedrock of the health care delivery system. Free health services are also being offered by most states and local governments across the country. All of these have resulted in a downward trend in the incidence/prevalence of cardiac diseases such as RHD and its underlying or associated infectious etiology. At LTH, a grossly low prevalence of $0.002 \%$ and $0.004 \%$ for endomyocardial fibrosis in cardiac and medical patients, respectively, was recently reported compared to a prevalence of $10 \%$ that was obtained in the 1960s and $1970 \mathrm{~s}$ in Nigeria. ${ }^{22}$ Endomyocardial fibrosis is another disease presumed to be indirectly linked with infectious agents.

The majority of the patients (70\%) in the current study were aged 18-24 years, an age that is the entry point into the reproductive years, ie, when most women are likely to be carrying their first pregnancy. This is in keeping with the reviews done in South Africa where the majority (over 50\%) of patients presenting were younger than 25 years. ${ }^{6,11}$ However, a Canadian study reported the median age for presentation with cardiac disease during pregnancy to be 32 years. ${ }^{23}$ In another review of various studies pooled from 28 countries with over 60 hospitals, the median age was 30 years. The much lower age of presentation in Africa may be due to the young 


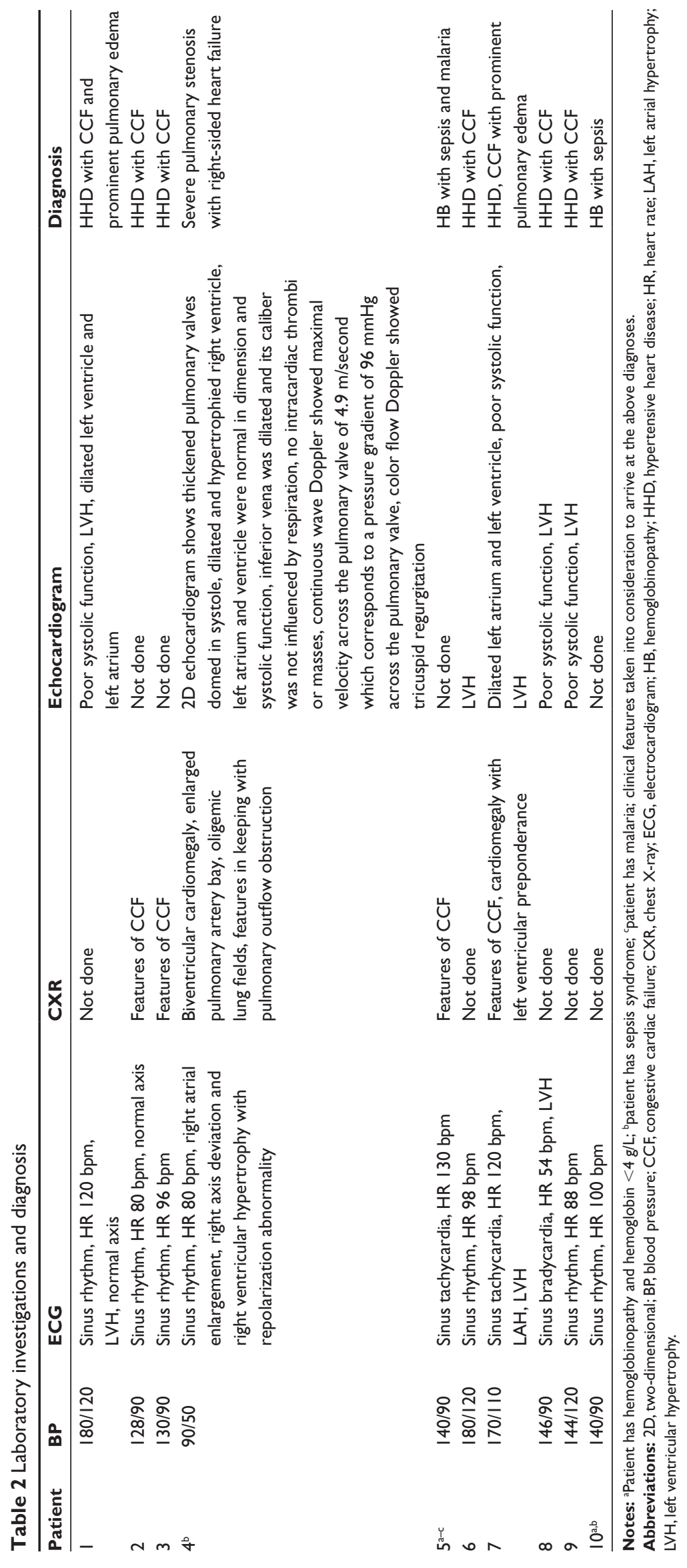


Table 3 Treatment of study patients

\begin{tabular}{lll}
\hline Patient & Diagnosis & Treatment \\
\hline I & HHD with CCF and pulmonary & Hydralazine, $\alpha$-methyldopa, digoxin, furosemide, intranasal oxygen, warfarin (postdelivery) \\
2 & edema & Furosemide, aminophylline, intranasal oxygen \\
3 & HHD with CCF & Furosemide, aminophylline, intranasal oxygen \\
4 & HHD with CCF & Furosemide, spironolactone, warfarin (postdelivery), amoxicillin-clavulanic acid, intranasal oxygen, \\
& Pulmonary stenosis & blood transfusion postdelivery \\
5 & HB with sepsis & Intranasal oxygen, cefuroxime, metronidazole, furosemide, antimalarial, intranasal oxygen, blood \\
6 & transfusion \\
7 & HHD with CCF and prominent & Fydralazine, $\alpha$-methyldopa, furosemide, intranasal oxygen \\
& Pulmonary edema & Furosemide, $\alpha$-methyldopa, intranasal oxygen, spironolactone, amoxicillin-clavulanic acid, warfarin \\
9 & HHD with CCF & Furosemide, aminophylline, intranasal oxygen \\
I0 & HBD with CCF & Furosemide, spironolactone, digoxin, intranasal oxygen \\
\hline
\end{tabular}

Abbreviations: CCF, congestive cardiac failure; HB, hemoglobinopathy; HHD, hypertensive heart disease.

age at marriage and early commencement of child bearing. The first pregnancy is fraught with a lot of danger as this may unearth or aggravate an underlying medical or cardiac disease in an otherwise clinically stable nulliparous woman. Fifty percent of the patients in the current study were primigravidae, which further confirms that the first pregnancy more often reveals the underlying cardiac condition. However, it is worth mentioning that two studies from South Africa reported a higher incidence of 53\% and $65.6 \%$ respectively. ${ }^{6,11}$ The three neonatal/perinatal deaths occurred in the babies of women aged 18-24 years who were also primigravidae, ie, the same groups in which the two maternal deaths occurred. This further underscores that a young primigravid woman is a high-risk patient. Other factors contributing to these high neonatal and maternal mortalities were failure to register for antenatal care until the point of presentation with heart failure during pregnancy and lack of prepregnancy screening to identify these cohorts of patients with subclinical cardiac disease. All of the patients were unbooked, which is also in keeping with a study revealing that there were more occurrences of maternal and fetal/perinatal morbidity and mortality in the unbooked primigravidae. ${ }^{24} \mathrm{~A} 10$-year review of maternal mortality in Enugu, Nigeria also reported that unbooked patients had a mortality rate 48 times higher than booked patients. ${ }^{25}$

It is also not unexpected that all of the patients presented between the second and third trimester as this period coincides with the period of a physiological increase in blood volume during pregnancy when cardiorespiratory adaptations occur; in cardiac disease patients, there are increased symptoms and cardiac decompensation. ${ }^{26}$ All the maternal and neonatal deaths occurred in the second trimester at the onset of these physiological changes; if medical care was sought earlier, there could have been a better prognosis. The complication rate has been shown to be very low in pregnant women with pre-existing heart disease who received optimal antenatal care.

The two maternal deaths occurred in those with hemoglobinopathy and heart failure. Patients with hemoglobinopathy can have cardiomyopathy or hemochromatosis due to chronic blood transfusion. Moreover, the superimposed sepsis in these two patients with hemoglobinopathy could also have caused secondary cardiomyopathy due to multiorgan failure. Both of them had severe anemia with hemoglobin concentration less than $5 \mathrm{~g} / \mathrm{dL}$. Patients with an acute event associated with falling hemoglobin (less than $5 \mathrm{~g} / \mathrm{dL}$ ) can die suddenly from cardiovascular collapse. ${ }^{27}$ Anemia can also cause high output heart failure. All of these conditions stated above would have culminated in the congestive cardiac failure with which these two patients presented. Patients with hemoglobinopathy cope poorly with pregnancy for many reasons such as anemia, the different types of crisis associated with this disease, and the superimposed hemodynamic changes of pregnancy. Fetal death also occurred in this group. This is not uncommon as severe anemia during pregnancy has been associated with increased risk of prematurity, spontaneous abortion, low birth weight, intrauterine growth retardation, and fetal death. ${ }^{27,28}$

Hypertensive heart failure accounted for $70 \%$ of all the cases and six of the seven patients complied with their inhospital treatment and had favorable maternal and neonatal outcome, with one of them delivering twins. However, the seventh patient discharged herself against medical advice and was lost to follow-up. Five of the seven patients had left ventricular hypertrophy (Table 2). Heart failure and 
left ventricular hypertrophy are recognized as the most typical cardiac complications of hypertension and feature prominently in the newly proposed clinical classification of hypertensive heart disease by the working group of the Spanish Society of Cardiology. ${ }^{29}$ Hypertensive heart disease has been defined as a constellation of abnormalities that includes left ventricular hypertrophy and systolic and diastolic dysfunction; their clinical manifestations include arrhythmias and symptomatic heart failure. ${ }^{30}$ The prevalence of hypertension in Nigeria continues to increase. Its current prevalence in a semi-urban community in the same state (Osun State) as LTH is 36.6\% (using the Seventh Report of the Joint National Committee on Prevention, Detection, Evaluation, and Treatment of High Blood Pressure-based blood pressure threshold of $140 / 90 \mathrm{mmHg}$ ) or $13.3 \%$ (using a blood pressure threshold of $160 / 95 \mathrm{mmHg}^{31}$ compared to the previous crude national prevalence of $11.2 \%$ using the World Health Organization-based blood pressure threshold of $160 / 95 \mathrm{mmHg} .{ }^{32}$ Hence, a corresponding increase is expected not only in the prevalence of hypertension during pregnancy but also that of hypertensive heart failure. Two of the patients presented with acute pulmonary edema and another two had proteinuria; however, they all responded favorably to treatment and had good neonatal and maternal outcome.

Only one case of CHD was seen in the current study. She presented with rare pulmonary stenosis and right-sided heart failure and although she survived, she had a bad neonatal outcome (early perinatal death) - delivering her baby within 2 days of admission. This patient had severe pulmonary stenosis (Table 2) and needed percutaneous valvuloplasty to relieve the obstruction, but there is no facility for this in Nigeria. This procedure can be accommodated safely during pregnancy. ${ }^{33}$

All of the patients in the current study presented in NYHA classes III and IV in equal proportions. NYHA IV is a very bad prognostic index, hence it is not surprising that all the maternal and neonatal deaths occurred in patients in this class. In a study in sub-Saharan Africa, maternal death was significantly associated with NYHA functional class III or IV $(P=0.001) .{ }^{18}$ Heart disease during pregnancy accounts for a 100-fold higher maternal mortality compared with normal pregnancy in London, UK. Poor fetal outcome correlates closely with NYHA class, ${ }^{34}$ hence women with heart failure in a poor functional class should be encouraged to avoid pregnancy. Recently, the European Registry on Pregnancy and Heart Disease found that the majority of these patients go safely through pregnancy; however, pregnancy outcomes were markedly worse in patients with cardiomyopathy and in developing countries. ${ }^{23}$

A limitation of this study is its retrospective nature, which did not allow for screening of all pregnant women for heart disease. Hence, the cases of all those who presented with symptomatic heart failure for cardiac disease were reviewed. A community-based prospective study is encouraged to screen pregnant women for cardiac disease in order to have a clearer epidemiologic picture of this disease during pregnancy. This will serve as a baseline for planning towards the reduction/elimination of maternal mortality, which is one of the Millennium Development Goals.

\section{Conclusion}

Data from this hospital-based review shows that heart failure during pregnancy in Nigeria is uncommon. However, there is a lack of data from other health facilities in Nigeria. All the patients in this study were unbooked for antenatal care in the authors' center. The highest maternal and fetal/perinatal mortality occurred in primigravid women, which further emphasizes the importance of early booking for antenatal care as well as a complete cardiovascular evaluation during the first antenatal care visit.

\section{Acknowledgments}

The authors express their sincere gratitude to Mr Asa and his team at the medical records department of LTH, Osogbo. The authors funded the study.

\section{Disclosure}

The authors report no conflicts of interest in this work.

\section{References}

1. Almange C. Pregnancy and congenital heart disease. Arch Mal Coeur Vaiss. 2002;95(11):1040-1044. French.

2. Drenthen W, Pieper PG, Roos-Hesselink JW, et al. Outcome of pregnancy in women with congenital heart disease: a literature review. $\mathrm{J} \mathrm{Am} \mathrm{Coll}$ Cardiol. 2007;49(24):2303-2311.

3. Grewal J, Siu SC, Ross HJ, et al. Pregnancy outcomes in women with dilated cardiomyopathy. J Am Coll Cardiol. 2009;55(1): $45-52$.

4. Siu SC, Colman JM, Sorensen S, et al. Adverse neonatal and cardiac outcomes are more common in pregnant women with cardiac disease. Circulation. 2002;105(18):2179-2184.

5. Siu SC, Sermer M, Colman JM, et al. Prospective multicenter study of pregnancy outcomes in women with heart disease. Circulation. 2001;104(5):515-521.

6. Nqayana T, Moodley J, Naidoo DP. Cardiac disease in pregnancy. Cardiovasc J Afr. 2008;19(3):145-151.

7. Klein LL, Galan HL. Cardiac disease in pregnancy. Obstet Gynecol Clin North Am. 2004;31(2):429-459.

8. Van Mook WN, Peeters L. Severe cardiac disease in pregnancy, part II: impact of congenital and acquired cardiac disease during pregnancy. Curr Opin Crit Care. 2005;11(5):435-448. 
9. Anandaraja S, Kothari SS, Bahl VK. Management of valvular heart disease during pregnancy. Indian Heart J. 2005;57(2):101-108.

10. Roos-Hesselink JW, Ruys TP, Stein JI, et al. Outcome of pregnancy in patients with structural or ischaemic heart disease: results of a registry of the European Society of Cardiology. Eur Heart $J$. 2013;34(9):657-665

11. Soma-Pillay P, Macdonald AP, Mathivha TM, Bakker JL, Mackintosh MO. Cardiac disease in pregnancy: a 4-year audit at Pretoria Academic Hospital. S Afr Med J. 2008;98(7):553-556.

12. Reirmold SC, Rutherford JD. Clinical practice. Valvular heart disease in pregnancy. N Engl J Med. 2003;349(1):52-59.

13. Burwell CS, Metcalfe J. Congestive phenomena occurring in pregnant women with heart disease. Circulation. 1960;21:430-435.

14. Gelson E, Johnson M, Gatzoulis M, Uebing A. Cardiac disease in pregnancy. Part 1: congenital heart disease. Obstet Gynecol. 2007;9: $15-20$.

15. Sawhney H, Aggarwal N, Suri V, Vasishta K, Sharma Y, Grover A. Maternal and perinatal outcome in rheumatic heart disease. Int J Gynaecol Obstet. 2003;80(1):9-14.

16. Silwa K, Wilkinson D, Hansen C, et al. Spectrum of heart disease and risk factors in a black urban population in South Africa (the Heart of Soweto Study): a cohort study. Lancet. 2008;371(9616): 915-922.

17. Moszynski P. South Africa's rising maternal mortality is due to health system failures, says report. BMJ. 2011;343:d5089.

18. Diao M, Kane A, Ndiaye MB, et al. Pregnancy in women with heart disease in sub-Saharan Africa. Arch Cardiovasc Dis. 2011;104(6-7): 370-374.

19. Adebayo RA, Akinwusi PO, Balogun MO, et al. Two dimensional and Doppler echocardiographic evaluation of 2501 patients presenting at OAUTHC, Ile-Ife, Nigeria. West African College of Physicians Abstracts. 36th Annual General and Scientific Meeting. 2012 Nov 4-8; Accra, Republic of Ghana.

20. Ogah OS, Adegbite GD, Akinyemi RO, et al. Spectrum of heart diseases in a new cardiac service in Nigeria: an echocardiographic study of 1441 subjects in Abeokuta. BMC Res Notes. 2008;1:98.

21. Sani MU, Karaye KM, Borodo MM. Prevalence and pattern of rheumatic heart disease in the Nigerian savannah: an echocardiographic study. Cardiovasc J Afr. 2007;18(5):295-299.
22. Akinwusi PO, Odeyemi AO. The changing pattern of endomyocardial fibrosis in South-west Nigeria. Clin Med Insights Cardiol. 2012;6:163-168.

23. Silversides CK, Colman JM, Sermer M, Siu SC. Cardiac risk in pregnant women with rheumatic mitral stenosis. Am J Cardiol. 2003;91(11): 1382-1385.

24. Danish N, Fawad A, Abbasi N. Assessment of pregnancy outcome in primigravida: comparison between booked and unbooked patients. J Ayub Med Coll Abbottabad. 2010;22(2):23-25.

25. Chukudebelu WO, Ozumba BC. Maternal mortality at the University of Nigeria Teaching Hospital, Enugu: a 10-year survey. Trop J Obstet Gynaecol. 1988;1(1):23-26.

26. Davies GA, Herbert WN. Assessment and management of cardiac disease in pregnancy. J Obstet Gynaecol Can. 2007;29(4):331-336.

27. Green-top Guideline No 61: Management of Sickle Cell Disease in Pregnancy. London: Royal College of Obstetricians and Gynecologists; 2011. Available from: http://www.rcog.org.uk/files/rcog-corp/ GTG61_26082011.pdf. Accessed February 11, 2013.

28. Sifakis S, Pharmakides G. Anaemia in pregnancy. Ann N Y Acad Sci. 2000;900:125-136.

29. Alegria E, Gonzalez-Juanatey JR, Gonzalez-Maqueda I. Hypertensive heart disease: a new clinical classification (VIA). E-Journal of ESC Council for Cardiology Practice. 2009;7(20). Available from: http:// www.escardio.org/communities/councils/ccp/e-journal/volume7/ Pages/hypertensive-heart-disease-classification.aspx\#.URg_xqVwquI. Accessed February 7, 2012.

30. Drazner MH. The progression of hypertensive heart disease. Circulation. 2011;123(3):327-334

31. Adedoyin RA, Mbada CE, Balogun MO, et al. Prevalence and pattern of hypertension in a semiurban community in Nigeria. Eur J Cardiovasc Prev Rehabil. 2008;15(6):683-687.

32. National Expert Committee on Non-Communicable Diseases. Non-communicable Diseases in Nigeria. Final Report of a National Survey. Lagos: Federal Ministry of Health and Social Services; 1997.

33. Presbitero P, Prever SB, Brusca A. Interventional cardiology in pregnancy. Eur Heart J. 1996;17(2):182-188.

34. Martinez-Diaz JL. Valvular heart disease in pregnancy: a review of the literature. Bol Assoc Med P R. 2008;100(4):55-59.
International Journal of General Medicine

\section{Publish your work in this journal}

The International Journal of General Medicine is an international, peer-reviewed open-access journal that focuses on general and internal medicine, pathogenesis, epidemiology, diagnosis, monitoring and treatment protocols. The journal is characterized by the rapid reporting of reviews, original research and clinical studies across all disease areas.

\section{Dovepress}

A key focus is the elucidation of disease processes and management protocols resulting in improved outcomes for the patient. The manuscript management system is completely online and includes a very quick and fair peer-review system. Visit http://www.dovepress.com/ testimonials.php to read real quotes from published authors. 